\title{
UNA REGLA DE SINTONÍA NO ESTÁNDAR PARA CONTROLADORES PI POR EVENTOS
}

\author{
José Sánchez Moreno \\ Dpto. Informática y Automática, UNED, C/ Juan del Rosal 16, 28040 Madrid, jsanchez@dia.uned.es \\ María Guinaldo Losada \\ Dpto. Informática y Automática, UNED, C/ Juan del Rosal 16,28040 Madrid, mguinaldo@dia.uned.es \\ Sebastián Dormido Bencomo \\ Dpto. Informática y Automática, UNED, C/ Juan del Rosal 16, 28040 Madrid, sdormido@dia.uned.es
}

\begin{abstract}
Resumen
En este trabajo se presenta un método no estándar para la sintonización de un controlador PI basado en eventos. El principal objetivo principal de esta regla de sintonía es generar un conjunto de parámetros que introduzca al proceso en un ciclo límite en el que la frecuencia y amplitud hayan sido especificadas por el usuario. Mediante la introducción de un parámetro adicional, definido como margen de oscilación, el método se puede generalizar para producir parámetros que eviten los ciclos límite con cierto margen de robustez. Las simulaciones efectuadas demuestran la capacidad de la regla de sintonía para forzar al sistema a oscilar a una frecuencia y amplitud especificadas.
\end{abstract}

Palabras clave: Ciclo límite, eventos, sintonía, controlador PI.

\section{INTRODUCCIÓN}

La definición de un algoritmo de auto sintonía específicamente diseñado para un controlador PI basado en eventos conlleva dos pasos: (i) el desarrollo de un procedimiento que recurra a las características de muestreo basado en eventos para la estimación de los parámetros de una función de transferencia de primer o segundo orden que mimetice el comportamiento del proceso en una banda de frecuencias comprendida entre $\omega_{-180^{\circ}} \mathrm{y}$ $\omega_{-135^{\circ}}$, y (ii) la formulación de una regla de sintonía para el controlador PI basado en eventos que se apoye en la función de transferencia identificada previamente (o algunas características del proceso en caso de que se conozcan).

Métodos específicos para la identificación de los parámetros de funciones de transferencia en lazos de control PI basados en eventos han sido propuestos en los últimos años en varias publicaciones. La primera investigación fue descrita en [1]. En ese trabajo, los parámetros del proceso se estiman considerando un ciclo límite generado por un controlador PI basado en eventos que previamente ha sido sintonizado; el método se basa en el uso de la función descriptiva (DF) del muestreador basado en eventos. Otros dos métodos se describen en [2,3]. Los métodos de estimación de estas dos aportaciones son diferentes al descrito en [1] ya que se basan en ajustar un modelo de primer orden formulado en el espacio de estados recurriendo a las características de la señal oscilatoria de la salida del proceso. Otros métodos específicos para la identificación en lazos de control basados en eventos se han publicado recientemente en [4,5]; ambos métodos se basan en recurrir al controlador para forzar un ciclo límite $\mathrm{y}$, una vez que el sistema oscila de forma estable, extraer información relevante de la señal de salida (frecuencia, amplitud, punto crítico, armónicos) para utilizarse en la estimación de los parámetros de un conjunto de funciones de transferencia.

En lo que respecta a la sintonía de controladores PI basados en eventos, hay trabajos que aplican reglas de ajuste concebidas originalmente para controladores PI que operan en tiempo continuo como, por ejemplo, las reglas AMIGO o la SIMC. Algunas de estas contribuciones son Vasyutynskyy, y Kabitzsch [6], Beschi et al. [2,3,7], Pawlowski et al. [8] y Romero et al. [9]. El primer trabajo en el que se proponen reglas de sintonía ad-hoc para controladores PI basados en eventos y sistemas de primer orden con retardo (FOPTD) es en Beschi et al. [10]; una versión extendida de este trabajo se puede consultar en [11]. Otros trabajos recientes sobre procedimientos específicos para la sintonía de controladores PI basados en eventos se pueden consultar en Romero y Sanchís [12] y Ruiz et al. [13].

En este trabajo se presenta una regla de sintonía con una finalidad completamente diferente y opuesta a las reglas de sintonía clásicas. De hecho, el objetivo principal de esta nueva regla es generar un conjunto de parámetros para un controlador PI por eventos que fuercen al proceso a oscilar a una frecuencia (o tiempo entre eventos) y amplitud pre-fijadas por el usuario. El objetivo final es proporcionar una forma 
supervisada de obligar al sistema a oscilar para, por ejemplo, periódicamente volver a identificar los parámetros de la función de transferencia del proceso (una especie de enfoque de detección de fallos basada en eventos). Sin embargo, este método no estándar se puede generalizar mediante la introducción de un parámetro adicional que permita a la regla de sintonía comportarse como una regla estándar. Fijando este nuevo parámetro a cero, la regla de sintonía se comportaría como una regla clásica.

El trabajo se organiza de la siguiente forma. En la Sección 2 se presenta la arquitectura de un sistema de control por eventos y se explica de forma breve la razón de la aparición de ciclos límites en este tipo de sistemas. En la Sección 3 se describe la regla de sintonía basada en la especificación de la frecuencia de oscilación con una amplitud mínima. En la Sección 4 se modifica la regla para poder especificar la amplitud de la oscilación. La generalización de la regla mediante la inclusión de un parámetro conocido como margen de oscilación se describe en la Sección 5. El artículo finaliza con unas breves conclusiones y posibles líneas de continuación.

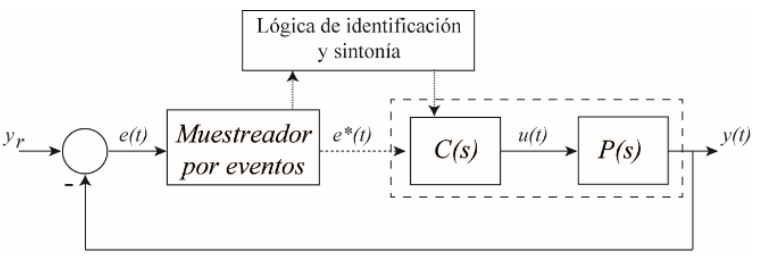

Figura 1: Esquema de control basado en eventos.

\section{SISTEMA DE CONTROL PI BASADO EN EVENTOS}

La arquitectura de control considerada en este trabajo se presenta en la Figura 1. En este sistema de control basado en eventos, cuando el muestreador detecta un evento transmite la información al controlador $C(s)$, que en este caso se considera que es un PI. Para forzar el disparo de un evento se han propuesto en la literatura diferentes condiciones lógicas o de disparo. La condición que se considera aquí se denomina send-on-delta simétrico (SSOD) [4], que no es más que un caso especial del conocido método send-ondelta (SOD), también denominado deadband sampling. Matemáticamente, su comportamiento se describe como

$e^{*}(t)=$
$\begin{cases}(i+1) \delta & \text { if } e(t) \geq(i+1) \delta \wedge e^{*}\left(t^{-}\right)=i \delta \\ i \delta & \text { if } e(t) \in[(i-1) \delta,(i+1) \delta] \wedge e^{*}\left(t^{-}\right)=i \delta \\ (i-1) \delta & \text { if } e(t) \leq(i-1) \delta \wedge e^{*}\left(t^{-}\right)=i \delta\end{cases}$
Mediante esta condición de disparo, el muestreador recibe una señal continua $e(t)$ y genera una señal muestreada $e^{*}(t)$ que es múltiplo de $\delta$. La clave de la relación entre $e(t)$ y $e^{*}(t)$ es que ésta se puede considerar como una generalización de un relé con histéresis. Esto implica que una función descriptiva de esta no linealidad puede derivarse y aplicarse al análisis de las oscilaciones [14].

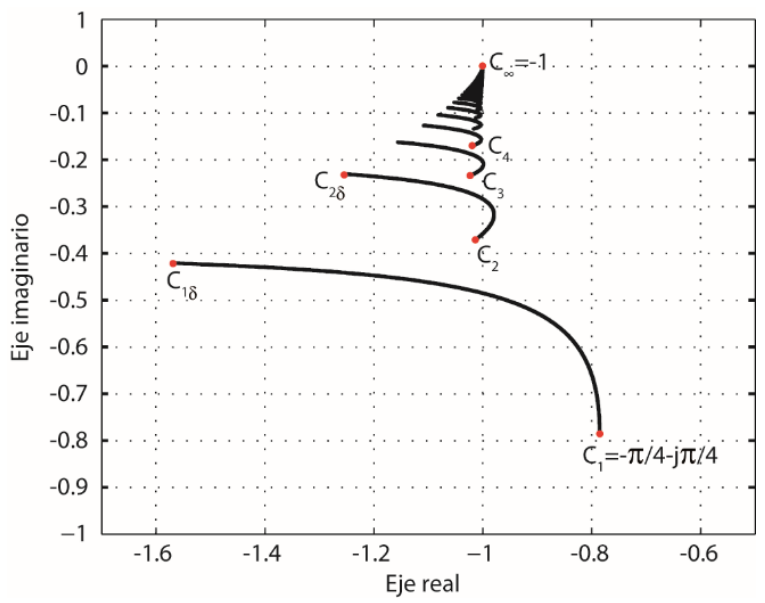

Figura 2: Mapa de Nyquist de $-1 / N(A, \delta)$.

La función descriptiva del muestreador SSOD viene dada por la siguiente expresión [9]

$$
\begin{aligned}
& N(A, \delta)= \\
& \frac{2 \delta}{\pi A}\left[1+\sqrt{1-\left(\frac{\delta}{A} m\right)^{2}}+2 \sum_{k=1}^{m-1} \sqrt{1-\left(\frac{\delta}{A} k\right)^{2}}\right] \\
& -j \frac{2 \delta^{2}}{\pi A^{2}} m
\end{aligned}
$$

donde $A$ es la amplitud de la señal sinusoidal de entrada y $m=\lfloor A / \delta\rfloor$. Una representación gráfica de $-1 / N(A, \delta)$ se muestra en la Figura 2 para $A \in[\delta, \infty)$. Cada intersección del sistema $G(s)=C(s) P(s)$ con un arco de los que componen la representación de $-1 / N(A, \delta)$ produce una oscilación, o ciclo límite, de amplitud y frecuencia diferentes. Intersecciones del sistema con el arco que comienza en el punto $C_{1}$ $\mathrm{y}$ concluye en el $C_{1 \delta}$ producen oscilaciones de amplitudes $A \in[\delta, 2 \delta)$, intersecciones con el arco que comienza en $C_{2}$ y termina en $C_{2 \delta}$ generan oscilaciones con $A \in[2 \delta, 3 \delta)$, y así sucesivamente.

Por ejemplo, la intersección de $G(s)$ con el punto $C_{1}=-\pi / 4-j \pi / 4$ en el plano de Nyquist representa $(1)$ la existencia de un ciclo límite con la mínima 
amplitud posible $A=\delta$ y frecuencia $\omega_{\text {osc }}$; esta frecuencia satisface la expresión $G\left(j \omega_{o s c}\right)=C_{1}$.

\section{SINTONÍA BASADA EN FRECUENCIA CON UNA AMPLITUD MÍNIMA}

Tal y como se ha explicado en el apartado previo, debido a la naturaleza de un sistema de control basado en eventos, hay dos posibles pero opuestas estrategias para la sintonización de los parámetros del controlador, en este caso un PI: (a) el método estándar para regulación y rechazo de perturbaciones y (b) un nuevo enfoque que fuerce al sistema a entrar en un ciclo límite con un amplitud y frecuencia definidas por el usuario. El objetivo de este nuevo enfoque no estándar, y en cierta forma contra intuitivo, es que, en algunas situaciones (fases de mantenimiento, comprobación de los actuadores, estudio de las características de los sensores) puede ser útil forzar al sistema a que oscile de forma controlada a una amplitud $y$ frecuencia preespecificadas gracias a la introducción de un nuevo conjunto de parámetros del controlador PI, no mediante la aplicación de acciones manuales introducidas directamente por un operador.

Las especificaciones para la sintonía del controlador recurriendo a este nuevo enfoque se reducen a indicar la frecuencia $\omega_{o s c}$ para que $G(s)$ oscile a esa frecuencia con la amplitud mínima posible, esto es $A=\delta$, utilizando la información proporcionada por el modelo de primer o segundo orden previamente identificado $\hat{P}(s)$ o, si se conoce, la función de transferencia del proceso real $P(s)$.

Como ya es sabido, un ciclo límite con frecuencia $\omega_{\text {osc }} \mathrm{y}$ amplitud $\delta$ se obtiene por la intersección de $G\left(j \omega_{o s c}\right)=P\left(j \omega_{o s c}\right) C\left(j \omega_{o s c}\right)$ con el punto crítico $C=-1 / N(\delta, \delta)$. Según (2), el punto $C$ es $-\pi / 4-j \pi / 4$. Sin embargo, dicho valor es una aproximación basada en la función descriptiva del muestreador SSOD y no tiene en cuenta el efecto de los armónicos más altos. Según [9], el punto exacto $C^{\prime}$ que define la condición de oscilación es

$$
\begin{aligned}
C_{r e}^{\prime} & =-\frac{\pi}{4}+\frac{1}{3} G_{r e}\left(j 3 \omega_{o s c}\right)-\frac{1}{5} G_{r e}\left(j 5 \omega_{o s c}\right) \\
& +\frac{1}{7} G_{r e}\left(j 7 \omega_{o s c}\right)-\ldots . \\
C_{i m}^{\prime} & =-\frac{\pi}{4}-\frac{1}{3} G_{i m}\left(j 3 \omega_{o s c}\right)-\frac{1}{5} G_{i m}\left(j 5 \omega_{o s c}\right) \\
& -\frac{1}{7} G_{i m}\left(j 7 \omega_{o s c}\right)-\ldots
\end{aligned}
$$

Desafortunadamente, este punto $C^{\prime}$ no se puede calcular ya que la expresión (3) considera infinitos armónicos del proceso real, cuya expresión se supone desconocida. Sin embargo, se puede derivar una aproximación ignorando, por ejemplo, los armónicos superiores a 7 y utilizando el modelo $\hat{P}(s)$. Esta aproximación se define como

$$
\begin{aligned}
\hat{C}_{r e}^{\prime} & =-\frac{\pi}{4}+\frac{1}{3} \hat{G}_{r e}\left(j 3 \omega_{o s c}\right)-\frac{1}{5} \hat{G}_{r e}\left(j 5 \omega_{o s c}\right) \\
& +\frac{1}{7} \hat{G}_{r e}\left(j 7 \omega_{o s c}\right) \\
\hat{C}_{i m}^{\prime} & =-\frac{\pi}{4}-\frac{1}{3} \hat{G}_{i m}\left(j 3 \omega_{o s c}\right)-\frac{1}{5} \hat{G}_{i m}\left(j 5 \omega_{o s c}\right) \\
& -\frac{1}{7} \hat{G}_{i m}\left(j 7 \omega_{o s c}\right)
\end{aligned}
$$

donde

$$
\hat{G}\left(j n \omega_{o s c}\right)=K_{p}\left(1+\frac{1}{j n \omega_{o s c} T_{i}}\right) \hat{P}\left(j n \omega_{o s c}\right) \quad n=1,3,5
$$

Como el punto crítico que produce el límite ciclo se define por

$$
\hat{G}\left(j \omega_{o s c}\right)=K_{p}\left(1+\frac{1}{j \omega_{o s c} T_{i}}\right) \hat{P}\left(j \omega_{o s c}\right),
$$

igualándolo con (4), es posible plantear el siguiente sistema

$$
\begin{aligned}
& \hat{G}_{r e}\left(j \omega_{o s c}\right)=\hat{C}_{r e}^{\prime} \\
& \hat{G}_{i m}\left(j \omega_{o s c}\right)=\hat{C}_{i m}^{\prime} .
\end{aligned}
$$

Como los parámetros del modelo son conocidos, el sistema (7) es lineal, las expresiones para $K_{p}$ y $T_{i}$ se pueden obtener recurriendo a una herramienta de cálculo simbólico. En aras de la brevedad, estas expresiones no se incluyen en este documento. Si la función de transferencia exacta del proceso real se conociese, las expresiones de (4) a (7) sería similares pero utilizando $P(j \omega)$.

Ejemplo 1: Sea el proceso $P(s)=(s+1)^{-4}$ y su modelo $\hat{P}(s)=1.003 e^{-1.945 s} /(2.503 s+1)$. Si el controlador PI se sintoniza para generar oscilaciones a $\omega_{\text {osc }}=0.3$ recurriendo a $\hat{P}(s)$ y fijando $\delta=1$, los parámetros obtenidos tras resolver (7) son $\left[K_{p}=0.659, T_{i}=1.586\right]$. En una simulación con estos parámetros, se observa que el sistema alcanza 
un ciclo límite de amplitud $\hat{A}=1.0003$ a $\hat{\omega}_{\text {osc }}=0.285$, muy cerca de las especificaciones iniciales (Figura 3). Los diagramas de Nyquist de $C(s) \hat{P}(s)$ and $C(s) P(s)$ se presentan en la Figura 4. Se puede observar las diferencias entre el punto $C$ de la DF, el punto $\hat{C}^{\prime}$ a $\omega_{\text {osc }}=0.3$ utilizado para calcular los parámetros del controlador y el punto donde la oscilación a $\hat{\omega}_{\text {osc }}=0.285$ realmente sucede si se utiliza el proceso real.

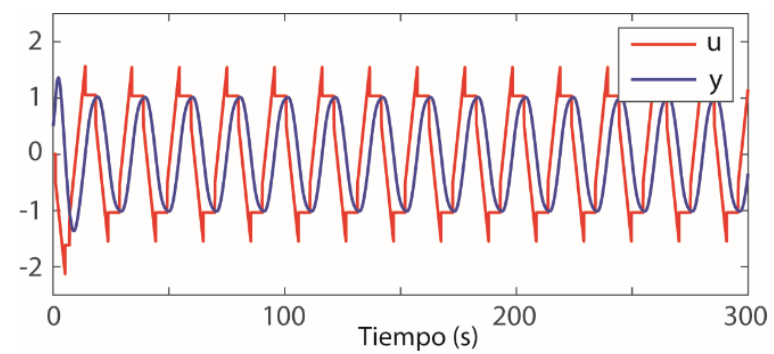

Figura 3: Ciclo límite de $P(s)=(s+1)^{-4}$ oscilando a $\hat{\omega}_{\text {osc }}=0.285$ con $\left[K_{p}=0.659, T_{i}=1.586\right]$.

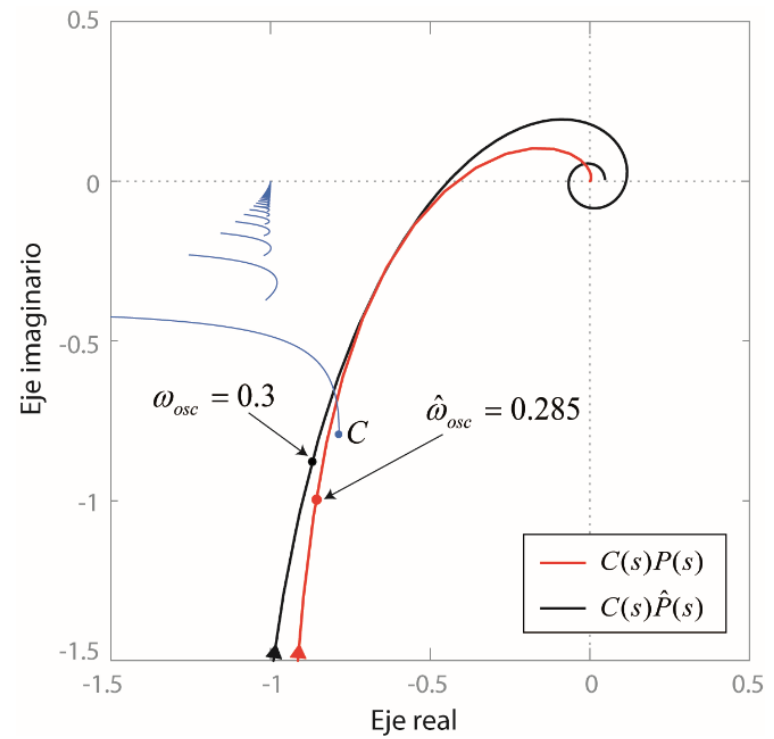

Figura 4: Diagramas de Nyquist de $C(s) \hat{P}(s)$ y $C(s) P(s)$ mostrando los diferentes puntos críticos.

Ahora, si el ajuste se realiza utilizando directamente el proceso $P(s)=(s+1)^{-4}$, los parámetros que generan un ciclo límite a $\omega_{o s c}=0.3$ son $\left[K_{p}=0.5132, T_{i}=1.3074\right] . \quad$ Realizando una simulación con estos parámetros, el sistema entra en un ciclo límite con $\hat{A}=1.014$ y $\hat{\omega}_{\text {osc }}=0.305$ (muy similar al representado en la Figura 3) que satisface las especificaciones de diseño.

\subsection{ANÁLISIS DEL RANGO DE FRECUENCIAS VÁLIDAS}

Ajustar el controlador para producir oscilaciones a ciertas frecuencias puede producir valores negativos de los parámetros del controlador. Por ejemplo, utilizando el proceso y el modelo del Ejemplo 1, los parámetros para generar un ciclo límite a $\omega_{\text {osc }}=0.03$ que se obtiene son $\left[K_{p}=-0.8937, T_{i}=-27.9612\right]$, produciendo un sistema estable de fase no mínima que oscila a $\hat{\omega}_{\text {osc }}=0.04$.

Así pues, parece adecuado conocer el rango de frecuencias de diseño en el que los parámetros del controlador que se obtienen son positivos. Debe observarse que, en general, parámetros del controlador positivos pueden producir inestabilidad en un lazo de control clásico. Sin embargo, en un sistema de control por eventos, los parámetros positivos siempre producen sistemas estables aplicando la regla de sintonía ya que está diseñada para provocar la intersección del sistema con un punto $\hat{C}^{\prime}$ que evita rodear el punto crítico -1 .

Analizar las expresiones de $K_{p}$ y $T_{i}$ derivadas de (7) resulta complicado. Una solución mucho más sencilla es obtener $K_{p}$ y $T_{i}$ recurriendo al punto téorico $C=-\pi / 4-j \pi / 4$ en lugar de su aproximación $\hat{C}^{\prime}$. Esto significa ignorar los armónicos en (7) y resolver

$$
\hat{G}\left(j \omega_{o s c}\right)=C,
$$

donde $\hat{G}\left(j \omega_{o s c}\right)$ viene dado por (6). Sustituyendo (6) en (8) y resolviendo para $K_{p}$ y $T_{i}$ se obtiene,

$$
\begin{gathered}
K_{p}=-\frac{\pi\left(P_{r e}\left(j \omega_{o s c}\right)+P_{i m}\left(j \omega_{o s c}\right)\right)}{4\left(P_{r e}^{2}\left(j \omega_{o s c}\right)+P_{i m}^{2}\left(j \omega_{o s c}\right)\right)} \\
T_{i}=\frac{\left(P_{r e}\left(j \omega_{o s c}\right)+P_{i m}\left(j \omega_{o s c}\right)\right)}{\omega_{o s c}\left(P_{i m}\left(j \omega_{o s c}\right)-P_{r e}\left(j \omega_{o s c}\right)\right)}
\end{gathered}
$$

Analizando (9), el rango de $\omega_{\text {osc }}$ que produce un conjunto de parámetros positivos satisface

$$
\left|P_{i m}\left(j \omega_{o s c}\right)\right|>\left|P_{r e}\left(j \omega_{o s c}\right)\right| \text {. }
$$

Esto significa que el intervalo de frecuencias de diseño válidas está acotado por

$$
\begin{aligned}
& -\frac{\pi}{4}=\arg P\left(j \omega_{\text {osc_min }}\right) \\
& -\frac{3 \pi}{4}=\arg P\left(j \omega_{\text {osc_max }}\right)
\end{aligned} .
$$


Debe observarse que para procesos con integradores, $\omega_{\text {osc_min }}$ es cero.

Ejemplo 2: Continuando con el proceso y el modelo del Ejemplo 1, si $C(s)$ se diseña para conseguir $\omega_{\text {osc }}=0.03$ recurriendo a $\hat{P}(s)$, los parámetros que se obtienen recurriendo a la resolución de (7) son $\left[K_{p}=-0.8937, T_{i}=-27.9612\right]$ y el sistema resultante es estable de fase no mínima. Si la sintonía se realiza para oscilar a $\omega_{o s c}=1$, los parámetros son $\left[K_{p}=1.9634, T_{i}=-0.9787\right] \quad$ y el sistema es inestable. Aplicando (11) a $\hat{P}(s)=1.003 e^{-1.945 s} /(2.503 s+1)$, el rango de frecuencias válidas para sintonizar el controlador es $0.183<\omega_{\text {osc }}<0.678$. Este rango es coherente con los dos conjuntos de parámetros obtenidos en este ejemplo.

Ejemplo 3: Considérese el proceso

$$
P(s)=\frac{(-s+1) e^{-5 s}}{s(s+1)^{5}}
$$

identificado como

$$
\hat{P}(s)=\frac{1.0001 e^{-8.9984 s}}{s(2.0233 s+1)} .
$$

Aplicando la expresión (11), el rango de frecuencias donde es posible sintonizar el controlador PI con parámetros positivos para forzar un ciclo límite es $0<\omega_{\text {osc }}<0.07134$. Teniendo en cuenta este rango y sintonizando el controlador mediante la resolución de (5) para generar un ciclo límite a muy baja frecuencia $\omega_{\text {osc }}=0.04$, los parámetros que se obtienen son $\left[K_{p}=6.79322 e-04, T_{i}=8.4322 e+02\right] . \quad \mathrm{Si} \quad$ se realiza una simulación con estos parámetros, la frecuencia del ciclo límite resultante es $\hat{\omega}_{\text {osc }}=0.039$ y la amplitud es 1.012. La Figura 5 presenta el ciclo límite de $P(s)$ y los diagramas de Nyquist del proceso real y del modelo; debe observarse que el diagrama de Nyquist del sistema real se superpone con el correspondiente al modelo.

\section{SINTONÍA BASADA EN FRECUENCIA Y AMPLITUD}

Para el diseño de los parámetros del controlador con el fin de producir un ciclo límite de amplitud $A>\delta$ es necesario calcular el punto $C$ cuya intersección con el sistema en el diagrama de Nyquist produzca el efecto deseado. Este punto se puede aproximar mediante la expresión basada en el análisis DF del muestreador SSOD dado por (2) de la siguiente forma

$$
\begin{aligned}
& C_{r e}=\operatorname{Re}(-1 / N(A, \delta)) \\
& C_{i m}=\operatorname{Im}(-1 / N(A, \delta)),
\end{aligned}
$$

y resolviendo la expresión $\hat{G}\left(j \omega_{\text {osc }}\right)=C_{r e}+j C_{i m}$ para obtener los parámetros del controlador. El resultado es

$$
\begin{gathered}
K_{p}=-\frac{C_{r e} P_{r e}\left(j \omega_{o s c}\right)+C_{i m} P_{i m}\left(j \omega_{o s c}\right)}{P_{r e}^{2}\left(j \omega_{o s c}\right)+P_{i m}^{2}\left(j \omega_{o s c}\right)} \\
T_{i}=\frac{C_{r e} P_{r e}\left(j \omega_{o s c}\right)+C_{i m} P_{i m}\left(j \omega_{o s c}\right)}{\omega_{o s c}\left(C_{r e} P_{i m}\left(j \omega_{o s c}\right)-C_{i m} P_{r e}\left(j \omega_{o s c}\right)\right)}
\end{gathered}
$$

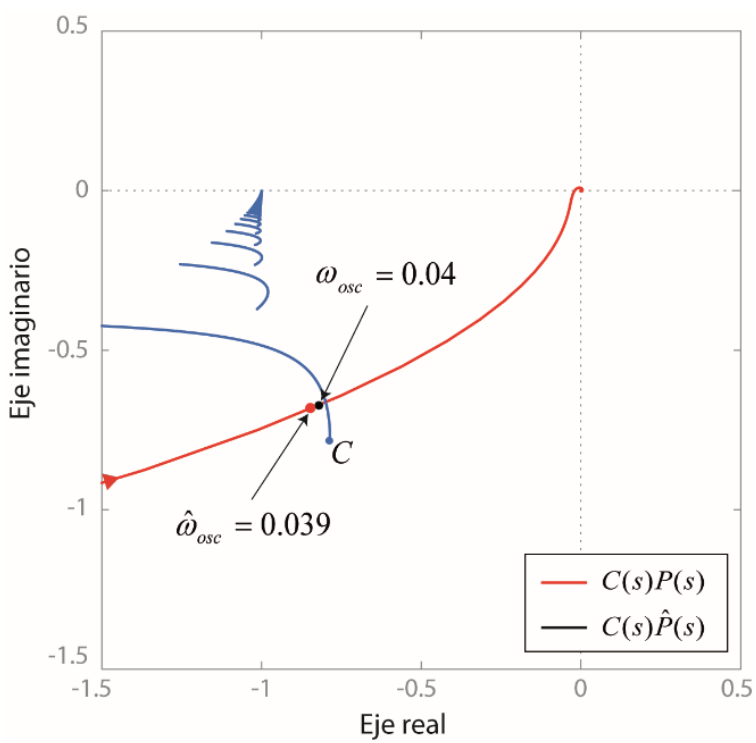

(b)

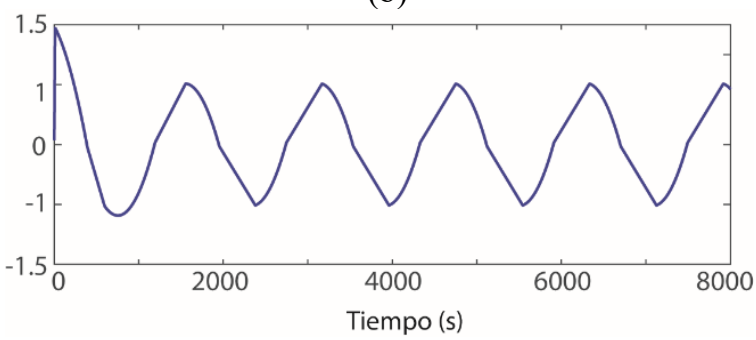

(a)

Figura 5: (a) Ciclo límite de

$P(s)=(-s+1) e^{-5 s} /\left[s(s+1)^{5}\right]$ a $\hat{\omega}_{o s c}=0.039 \mathrm{y}(\mathrm{b})$ diagramas de Nyquist del proceso real y del modelo.

Ejemplo 4: Continuando con el proceso $P(s)=(s+1)^{-4}$ y su modelo de primer orden 
$\hat{P}(s)=1.003 e^{-1.945 s} /(2.503 s+1), \quad$ si $\quad$ el controlador se sintoniza para producir un ciclo límite a $\omega_{\text {osc }}=0.3$ con $A=1.5$ utilizando $\hat{P}(s)$, los parámetros que se obtienen mediante la resolución de (39) y (40) son $\left[K_{p}=0.0341, T_{i}=0.0723\right]$. En una simulación de $P(s)$ con estos parámetros, se observa que sistema alcanza un ciclo límite de amplitud $\hat{A}=1.515$ a $\hat{\omega}_{\text {osc }}=0.312$ (véase la Figura 6). Sin embargo, si la sintonía del controlador se realiza recurriendo al proceso real $P(s)$, los parámetros son negativos, esto es, $\left[K_{p}=-0.0718, T_{i}=-0.16\right] \quad$ y el ciclo límite resultante tiene una amplitud $\hat{A}=1.56$ oscilando a $\hat{\omega}_{\text {osc }}=0.296$.

Como conclusión, para alcanzar el ciclo límite indicado en las especificaciones de sintonía, es necesario excitar el sistema con las condiciones adecuadas. En el ejemplo de la Figura 7, la perturbación aplicada a la salida del proceso para alcanzar los tres posibles ciclos límite sería $w=\{1.1,2.1,3.1\}$ considerando que $\delta=1$.

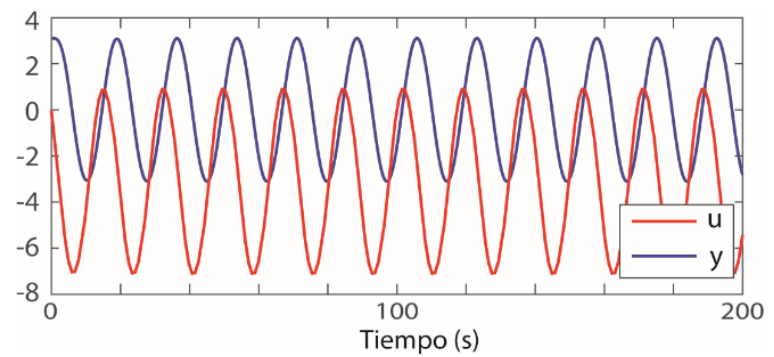

(b)

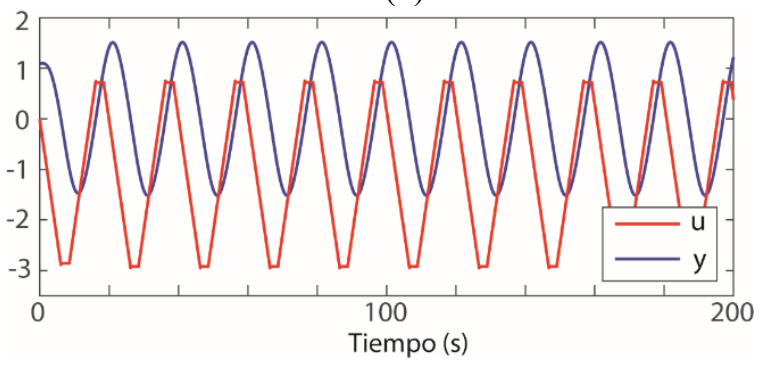

(a)

Figura 6: Simulación de $P(s)=(s+1)^{-4}$ con $\left[K_{p}=0.0341, T_{i}=0.0723\right]$ oscilando a (a) $\hat{A}=1.515$, $\hat{\omega}_{\text {osc }}=0.3125$ y (b) $\hat{A}=3.1, \hat{\omega}_{\text {osc }}=0.3619$.

Perturbaciones constantes $w$ de amplitud 1.1 (a) y 3.1 (b) se aplican a la salida del proceso para excitar el sistema y empezar a oscilar. En ambos casos, $\delta=1$.

Sin embargo, el análisis anterior no es completo debido a que los parámetros de control obtenidos de (13) pueden generar ciclos límite en diferentes frecuencias y amplitudes dependiendo de las condiciones iniciales que se apliquen al proceso para activar la respuesta del muestreador SSOD. Así, en el ejemplo anterior una perturbación de amplitud constante 1.1 permite obtener los resultados visualizados en la Figura 6.a, pero si el valor de la perturbación pasa a ser 3.1 , los parámetros del ciclo límite son $\hat{A}=3.1$ a $\hat{\omega}_{\text {osc }}=0.3619$ (Figura 6.b). Esto es consecuencia de que la intersección de la recíproca negativa de la DF con el sistema en el diagrama de Nyquist puede suceder en varios puntos (es decir, en diferentes frecuencias y amplitudes), de forma que la solución proporcionada por (13) es solo uno de los posibles puntos de intersección; converger en una u otra solución depende de las condiciones iniciales aplicadas al sistema. La Figura 7 presenta el diagrama de Nyquist de $\hat{P}(s)=1.003 e^{-1.945 s} /(2.503 s+1) \quad$ aplicando $\left[K_{p}=0.0341, T_{i}=0.0723\right]$ al controlador; se puede aprecia en la Figura 7 que, en función de las condiciones iniciales, el sistema podría converger a uno de los tres ciclos límite que resultan de la intersección del proceso y el muestreador.

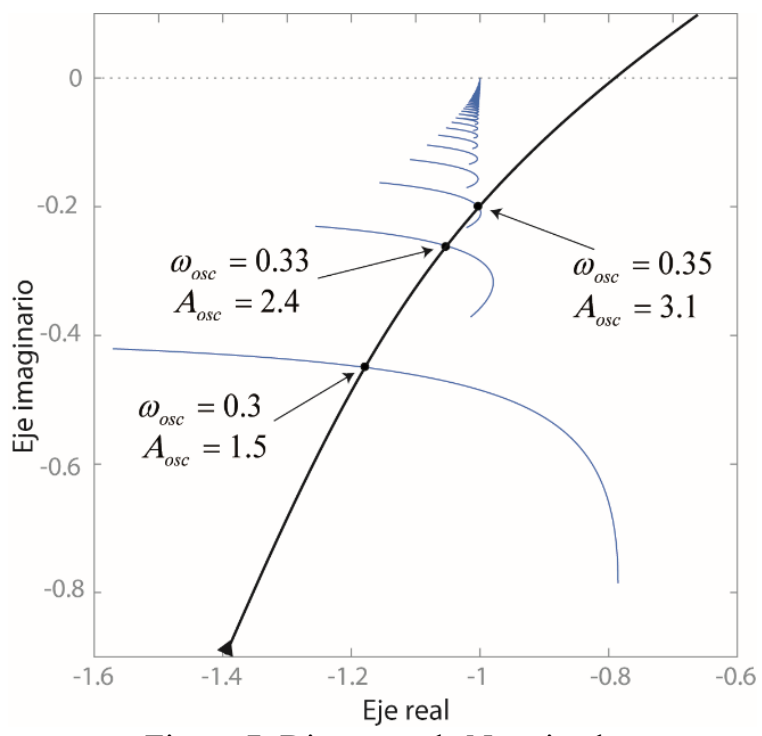

Figura 7: Diagrama de Nyquist de $\hat{P}(s)=1.0026 e^{-1.945 s} /(2.5032 s+1)$ con los parámetros $\left[K_{p}=0.0341, T_{i}=0.0723\right]$.

Al igual que se ha realizado para la sintonía basada en frecuencia a una amplitud mínima (Sección 3), es posible analizar el rango de frecuencias factible para obtener ciclos límite de amplitud superior a $\delta$ con parámetros de control positivos.

Obsérvese que utilizar puntos de $-1 / N(A, \delta)$ para alcanzar ciclos límite con amplitudes asociadas al 
extremo izquierdo de las curvas, por ejemplo, $A=1.99 \delta$, podría introducir inestabilidad si se llegase a rodear el punto crítico -1 . La recomendación es recurrir a amplitudes $A=n \delta$ con $n=1,2,3$.

\section{ADAPTANDO LA REGLA DE SINTONÍA PARA USO ESTÁNDAR}

Mantener el lazo de control oscilando durante largos períodos en la fase de mantenimiento implica un gasto innecesario de energía $\mathrm{y}$, al mismo tiempo, puede inducir un aumento del desgaste de los actuadores. Es por ello que, una vez se da por finalizada la fase de mantenimiento, el controlador debe volver a su estado de operación habitual. Un criterio aplicado habitualmente en la sintonía de un controlador PI es obtener unos parámetros de control que establezcan un margen de fase superior a $45^{\circ}$, tal y como se señala en $[9,12,15]$.

Continuando con esta idea, la regla de sintonía no estándar que se ha descrito previamente en (7), (9) y (13) puede generalizarse para aplicarse como una regla clásica para el seguimiento de referencias y rechazo de perturbaciones. Así, por ejemplo, teniendo en cuenta (7), el ciclo límite puede evitarse igualando $\hat{G}\left(j \omega_{o s c}\right)$, o $G\left(j \omega_{o s c}\right)$ en caso de que $P(s)$ sea conocido, a un punto separado de $\hat{C}^{\prime}$ por un cierto margen de oscilación $o_{m}$. Se tendría así

$$
\hat{G}\left(j \omega_{o s c}\right)=\left(1-o_{m}\right) \hat{C}^{\prime}
$$

donde $0 \leq o_{m}<1$. Si se selecciona un margen distinto de cero, entonces la resolución de (14) produciría un conjunto de parámetros que debería evitar las oscilaciones. De hecho, el valor de $o_{m}$ afecta, principalmente, a la ganancia proporcional ya que es la que produce un movimiento radial del punto crítico hacia el origen. Por ejemplo, considerando $o_{m}$ en (8),

$$
\hat{G}\left(j \omega_{o s c}\right)=\left(1-o_{m}\right) C
$$

y resolviendo, la expresión de $T_{i}$ no queda afectada por el margen de oscilación tal y como se puede apreciar

$$
\begin{aligned}
& K_{p}=-\frac{\pi\left(P_{r e}\left(j \omega_{o s c}\right)+P_{i m}\left(j \omega_{o s c}\right)\right)}{4\left(P_{r e}^{2}\left(j \omega_{o s c}\right)+P_{i m}^{2}\left(j \omega_{o s c}\right)\right)}\left(1-o_{m}\right) \\
& T_{i}=\frac{\left(P_{r e}\left(j \omega_{o s c}\right)+P_{i m}\left(j \omega_{o s c}\right)\right)}{\omega_{o s c}\left(P_{i m}\left(j \omega_{o s c}\right)-P_{r e}\left(j \omega_{o s c}\right)\right)}
\end{aligned}
$$

Este nuevo parámetro $o_{m}$ puede considerarse como un margen de ganancia desde el punto de vista de la DF del SSOD. En un sistema lineal, el margen de ganancia expresa cuánto se puede aumentar la ganancia del controlador antes de provocar oscilaciones. Y este parámetro representa exactamente lo mismo que viene a expresar en el modo lineal.

\section{CONCLUSIONES}

El método de sintonía que se ha presentado en este trabajo se ha concebido, principalmente, para conseguir forzar a un proceso a entrar en un ciclo límite a una frecuencia y amplitud definidas por el usuario. Es decir, la regla permite poner al sistema a oscilar de una forma estable y automática, sin necesidad recurrir a acciones manuales de control por parte de un operador. Mediante la introducción de un parámetro adicional, denominado margen de oscilación, el método puede comportarse como una regla se sintonía convencional. Los ejemplos de simulación que se han presentado demuestran la eficacia de esta nueva regla.

Algunos aspectos en los que continuar trabajando son el estudio de la inclusión de otro grado de libertad (por ejemplo, mediante la inclusión de un retardo de transporte) para conseguir alcanzar frecuencias bajas de oscilación con parámetros de control positivos.

\section{Agradecimientos}

Este trabajo ha sido financiado por el Ministerio de Economía y Competitividad mediante los proyectos DPI2014-55932-C2-2-R y DPI2017-84259-C2-2-R y por la Universidad Nacional de Educación a Distancia (UNED) mediante el proyecto 2018V/PREMIO/05.

\section{English summary}

\section{A NON-STANDARD TUNING RULE FOR EVENT-BASED PI CONTROLLERS}

\begin{abstract}
A new non-standard method for tuning an eventbased PI controller is presented. The main control objective of the new tuning rule is to generate a set of parameters that introduces the process in a limit cycle at a user-specified frequency and amplitude. By introducing an oscillation margin in the tuning rule,
\end{abstract}


the method generalizes to produce controller parameters that avoid limit cycles with certain robustness margin. Simulations demonstrate the effectiveness of the tuning rule to force the system to oscillate at a specified frequency and amplitude.

Keywords: Limit cycle, events, tuning, PI controller

\section{Referencias}

[1] Beschi, M., Dormido, S., Sánchez, J, Visioli, A., Yebra, L.J. (2014) "Event-based PI plus feedforward control strategies for a distributed solar collector field", IEEE Transactions on Control Systems Technology 22(4), pp. 1615-1622.

[2] Beschi, M., Dormido, S., Sánchez, J, Visioli, A. (2015) "Closed-loop automatic tuning technique for an event-based PI controller", Ind. Eng. Chem. Res. 54(24), pp. 6362-6370.

[3] Beschi, M., Dormido, S., Sánchez, J, Visioli, A. (2015) "An Event-based PI Controller Autotuning Technique for Integral Processes". $1^{\text {st }}$ IEEE International Conference on Event-Based Control, Communication, and Signal Processing (EBCCSP2015), Krakow (Poland)

[4] Sánchez, J., Guinaldo, M., Dormido, S., Visioli, A. (2018) "Identification of process transfer function parameters in event-based PI control loops", ISA Trans 75, pp. 157-171.

[5] Sánchez, J., Guinaldo, M., Dormido, S., Visioli, A. (2018) "Enhanced event based identification procedure for process control. Ind. Eng. Chem. Res. DOI: $10.1021 /$ acs.iecr.7b05239.

[6] Vasyutynskyy, V., Kabitzsch, K. (2006) "Implementation of PID controller with send-ondelta sampling". Proceedings of International Control Conference; Glasgow (UK).

[7] Beschi, M., Dormido, S., Sánchez, J., Visioli, A. (2012) "Characterization of Symmetric Send-onDelta PI Controllers”, Journal of Process Control 22, pp. 1930-1945.

[8] Pawlowski, A., Beschi, M., Guzmán, J.L., Visioli, A., Berenguel, M., Dormido, S. (2016) "Application of SSOD-PI and PI-SSOD Event-Based Controllers to Greenhouse Climatic Control", ISA Transactions, 65, pp. 525-536.

[9] Romero, J.A., Sanchís, R. (2016) “A New Method for Tuning PI Controllers with Symmetric Send-on-Delta Sampling Strategy", ISA Transactions 64, pp. 161-173.
[10] Beschi, M., Dormido, S., Sanchez, J., Visioli, A. (2012) "Tuning rules for event-based SSOD-PI controllers". Proceedings of the $20^{\text {th }}$ Mediterranean Conference on Control and Automation; Barcelona, (Spain).

[11] Beschi, M., Dormido, S., Sánchez, J., Visioli, A. (2014) "Tuning of Symmetric Send-on-delta Proportional-Integral Controllers", IET Control Theory \& Applications 8, pp. 248-259.

[12] Romero, J.A., Sanchís, R. (2018) "Tuning and robustness analysis of event-based PID controllers under different event generation strategies". International Journal of Control 91, pp. 1567-1587.

[13] Ruiz, A., Beschi, M., Visioli, A., Dormido, S. Jiménez, J.E. (2017) “A Unified Event-Based Control Approach for FOPTD and IPTD Processes Based on the Filtered Smith Predictor", Journal of the Franklin Institute 354, pp. 1239-1264.

[14] Gelb, A., Van der Velde, W.E. (1968) MultipleInput Describing Functions and Nonlinear System Design, McGraw-Hill, New York, USA.

[15] Åström, K.J., Hägglund, T. (2006) Advanced PID Control, ISA, 460 pages.

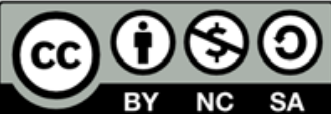

(C) 2019 by the authors. Submitted for possible open access publication under the terms and conditions of the Creative Commons Attribution CC BY-NC-SA 4.0 license (https://creativecommons.org/licenses/bync-sa/4.0/deed.es). 